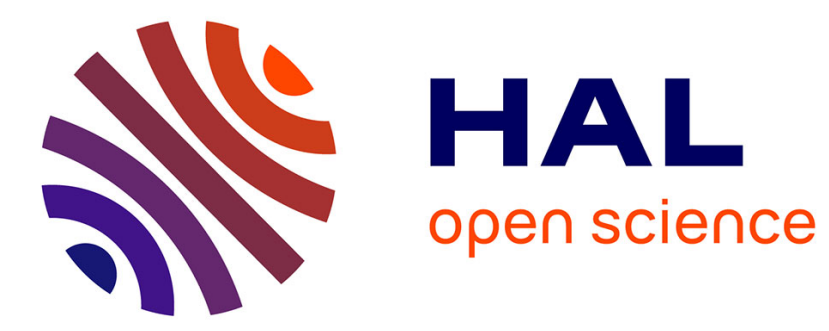

\title{
Trajectoires résidentielles familiales et interventions sociales à domicile
}

\author{
Bernadette Tillard, Anna Rurka
}

\section{To cite this version:}

Bernadette Tillard, Anna Rurka. Trajectoires résidentielles familiales et interventions sociales à domicile. Recherches familiales, 2013, 10, pp.75-89. 10.3917/rf.010.0075 . halshs-01228864

\section{HAL Id: halshs-01228864 \\ https://shs.hal.science/halshs-01228864}

Submitted on 14 Nov 2015

HAL is a multi-disciplinary open access archive for the deposit and dissemination of scientific research documents, whether they are published or not. The documents may come from teaching and research institutions in France or abroad, or from public or private research centers.
L'archive ouverte pluridisciplinaire HAL, est destinée au dépôt et à la diffusion de documents scientifiques de niveau recherche, publiés ou non, émanant des établissements d'enseignement et de recherche français ou étrangers, des laboratoires publics ou privés. 


\title{
TRAJECTOIRES RÉSIDENTIELLES FAMILIALES ET INTERVENTIONS SOCIALES À DOMICILE
}

\author{
Bernadette Tillard, Anna Rurka
}

\section{Union nationale des associations familiales (UNAF) | « Recherches familiales »}

2013/1 n $10 \mid$ pages 75 à 89

ISSN 1763-718X

Article disponible en ligne à l'adresse :

http://www.cairn.info/revue-recherches-familiales-2013-1-page-75.htm

\section{!Pour citer cet article :}

Bernadette Tillard, Anna Rurka, «Trajectoires résidentielles familiales et interventions sociales à domicile », Recherches familiales 2013/1 ( $\left.\mathrm{n}^{\circ} 10\right)$, p. 75-89.

DOI 10.3917/rf.010.0075

Distribution électronique Cairn.info pour Union nationale des associations familiales (UNAF).

(C) Union nationale des associations familiales (UNAF). Tous droits réservés pour tous pays.

La reproduction ou représentation de cet article, notamment par photocopie, n'est autorisée que dans les limites des conditions générales d'utilisation du site ou, le cas échéant, des conditions générales de la licence souscrite par votre établissement. Toute autre reproduction ou représentation, en tout ou partie, sous quelque forme et de quelque manière que ce soit, est interdite sauf accord préalable et écrit de l'éditeur, en dehors des cas prévus par la législation en vigueur en France. Il est précisé que son stockage dans une base de données est également interdit. 


\title{
T RaJeCtOIRES RÉSIDENTIELleS FAMILIALES ET INTERVENTIONS SOCIALES À DOMICILE
}

\author{
Bernadette Tillard, Anna Rurka
}

Dans le cadre d'une étude financée par l'ONED, nous avons réalisé 15 études de cas auprès de parents et de professionnels rencontrés à deux reprises, à un an d'intervalle. Ces familles avaient en commun de faire l'objet de plusieurs interventions dont au moins deux interventions simultanées dans leur espace domestique : celle d'un éducateur spécialisé dans le cadre d'une mesure éducative et celle d'une technicienne d'intervention sociale et familiale. La trajectoire résidentielle des familles et la sectorisation des services conduit à des incertitudes dans les interventions sociales. Elle crée une période de flottement durant laquelle la famille expérimente une certaine autonomie. Elle rend possible la recomposition des relations entre les parents et les travailleurs sociaux.

\section{$\checkmark$ Préoccupations sanitaires et socio-éducatives à l'egarard des enfants}

L'attention à la petite enfance s'est particulièrement développée au cours du XIX ${ }^{\mathrm{e}}$ siècle qui a donné lieu aux premières lois de protection de la mère et de l'enfant. La Troisième République n'a pas seulement rendu l'instruction obligatoire ${ }^{[1]}$, elle a également promulgué la loi Roussel et ainsi mis en place les premières mesures de santé publique qui tendent à protéger le nouveau-né et particulièrement l'enfant placé en nourrice ${ }^{[2]}$. Plus tard, après la Seconde Guerre mondiale, face au recul des indicateurs de santé périnatale, la création de la Protection maternelle et infantile (PMI ${ }^{[3]}$ a été une seconde phase de cette attention aux pratiques familiales de santé, mais également aux pratiques éducatives des familles, s'immisçant dans les pratiques quotidiennes d'hygiène, de soins et d'éveil de l'enfant.

Enfin, plus récemment, à la fin du $\mathrm{XX}^{\mathrm{e}}$ siècle, les besoins élémentaires des enfants étant couverts aussi bien sur le plan sanitaire qu'alimentaire, la préoccupation pour leur développement affectif et leur bien-être s'est développée, se manifestant en particulier au travers de la prévention

[1] Michèle GUIGUE, Bernadette TILLARD, «Parents et professionnels du travail éducatif : une relation en tension. Regards croisés autour de 20 jeunes en difficulté », Revue internationale de l'éducation familiale, n 27, 2010, pp. 57-81.

[2] Catherine ROLLET-ÉCHALIER. La politique à l'égard de la petite enfance sous la III République, Paris, INED, PUF, 1990.

[3] Alain NORVEZ. De la naissance à l'école. Santé, modes de garde et préscolarité dans la France contemporaine, Paris, PUFINED, 1990. 
de la maltraitance. Notre société tend progressivement à bannir les manifestations de violence physique. Contrairement au Canada, aux pays scandinaves et à certains pays de l'Europe de l'Est comme la Lettonie et la Pologne, à ce jour, il n'existe pas en France de loi interdisant toute forme de châtiments corporels comme la fessée. Cependant, toute correction physique de l'enfant est regardée avec suspicion et susceptible d'entraîner une immixtion des travailleurs sociaux dans l'intimité de la famille. L'avènement de l'enfant reconnu à la fois comme fragile et comme individu en devenir donne lieu à une attention particulière qui, après s'être manifestée par le placement institutionnel loin de son milieu familial, va laisser place à une politique de soutien à la parentalité qui privilégie le maintien de l'enfant au domicile familial ${ }^{[4]}$. L'aide éducative à domicile fait partie des modalités de soutien à la parentalitée ${ }^{[5]}$. Ces choix politiques s'inscrivent également dans une évolution des moyens financiers dont disposent l'État, puis les collectivités territoriales. Les placements institutionnels ont diminué au profit de l'aide éducative à domicile. Celle-ci prend la forme soit d'une Aide éducative en milieu ouvert (AEMO) prononcée par le juge des enfants, soit d'une Aide éducative à domicile (AED) relevant du service départemental d'Aide sociale à l'enfance (ASE).

Les préoccupations à la fois sanitaires et socio-éducatives de l'État à l'égard des enfants et l'organisation des différents services conduisent certaines familles à faire l'objet de plusieurs interventions simultanées. Notre étude soutenue par l'Observatoire national de l'enfance en danger (ONED) s'est intéressée à la manière dont les parents s'adaptent à la présence de différents travailleurs sociaux dont les interventions sont de nature différente, ainsi qu'aux collaborations et résistances à leurs sollicitations lorsque plusieurs interventions s'exercent au domicile familial ${ }^{[6]}$. Dans cet article, les résultats portant sur 15 études de cas seront analysés en mettant en 76 exergue les trajectoires résidentielles. Nous montrerons en quoi les déménagements interfèrent avec le suivi social des familles.

\section{\Méthode}

La recherche a porté sur des familles faisant l'objet de plusieurs interventions simultanées à leur domicile. Nous avions fixé comme critère d'inclusion que la famille reçoive au minimum un éducateur dans le cadre d'une mesure éducative et un technicien d'intervention sociale et familiale (TISF), quel que soit le cadre institutionnel de son intervention. Notre souci était d'inclure à la fois des modalités de travail s'appuyant essentiellement sur la rencontre régulière et la parole (AEMO 14 cas, AED 1 cas) et d'autres s'inscrivant davantage dans les préoccupations quotidiennes des familles et la participation aux activités domes-

[4] Bernadette TILLARD, Anna RURKA, Du placement à la suppléance familiale. Actualité des recherches internationales, Paris, L'Harmattan, 2009.

[5] David PIOLI, « Le soutien à la parentalité : entre émancipation et contrôle », Sociétés et jeunesses en difficulté, $\mathrm{n}^{\circ}$ 1, printemps 2006, 2006, http://sejed.revues.org/index106.html

[6] Anna RURKA, Bernadette TILLARD, Bernard VALLÉRIE, Emmanuelle MARTINS, Les interventions socio-éducatives en milieu ordinaire auprès de familles aux besoins multiples, Rapport final à l'ONED, 83 p., 2011.

[7] Dans la suite du texte, n'ayant rencontré que des femmes exerçant cette profession, nous emploierons le féminin.

[8] Anna RURKA, « Les parents face aux travailleurs sociaux. Quelles représentations pour quelle efficacité de l'intervention? » Les sciences de l'éducation - Pour l'Ère nouvelle, n 1, vol. 40, CERSE, Université de Caen, pp. 97-113, 2006 ; Patrick ROUSSEAU, Pratique des écrits et écriture des pratiques. La part « indicible » du métier d'éducateur, Paris, L'Harmattan, 2007. 
tiques (TISF) ${ }^{[7]}$. En effet, l'éducateur d'AEMO ${ }^{[8]}$ intervient dans la famille avec un mandat du juge des enfants pour un ou plusieurs enfants de la fratrie. Son intervention est centrée sur l'enfant mais, pour assurer le bien-être de l'enfant, son intervention s'adresse également $\mathrm{au}(\mathrm{x})$ parent(s). Son rôle est de leur apporter un soutien éducatif afin que les besoins de l'enfant soient pris en considération et que les relations avec les autres partenaires de l'éducation de l'enfant soient facilitées. Il peut ainsi susciter une inscription dans des activités de loisirs, veiller à ce que les parents assurent à l'enfant un environnement stable, sécurisé et adapté à son âge, accompagner une prise en charge médicale de l'enfant ou conseiller les parents dans leurs stratégies éducatives. Il rend compte de l'évolution de l'enfant et des relations familiales au juge des enfants afin de lui permettre de poursuivre, de modifier ou d'interrompre la prise en charge. Généralement, l'éducateur d'AEMO voit les parents une fois tous les quinze jours, gardant éventuellement le contact entre temps par un appel téléphonique. La TISF intervient le plus souvent à la demande des services sociaux (CAF, département, AEMO) afin de mettre en œuvre les objectifs de la prise en charge. Qu'elle soit directement employée par le service d'AEMO ou par une association d'aide à domicile, son intervention a lieu au domicile des familles durant des périodes plus longues (souvent 4 h), au moins une fois par semaine. Elle participe à toutes les tâches domestiques et éducatives ${ }^{[9]}$. Son travail comme celui de l'AEMO est centré sur l'enfant, mais il prend la forme d'un accompagnement des parents dans toutes les tâches nécessaires aux besoins de l'enfant. Aussi participe-t-elle à l'entretien de la maison, à l'approvisionnement, autant qu'aux démarches éducatives. Parfois, les travailleurs sociaux délèguent à ce professionnel de proximité des tâches de coordination ou d'accompagnement des parents relevant habituellement du rôle de l'éducateur ou de l'assistant social. « Faire avec » les parents leurs tâches ordinaires ou plus exceptionnelles permet à ces professionnelles d'avoir une place privilégiée auprès des parents et particulièrement de la mère. Cette proximité se manifeste volontiers dans les échanges informels entre familles et professionnelles ${ }^{[10]}$. L'intervention de la TISF peut être soit mentionnée dans l'ordonnance du juge, soit proposée par les services sociaux. Dans un cas comme dans l'autre, elle peut être effectuée soit par une association externe au service d'AEMO (12 cas sur 15), soit effectuée par une TISF employée par le service d'AEMO (3 cas sur 15). Lorsque l'intervention de la TISF est recommandée et que le service d'AEMO n'emploie pas de TISF, l'intervention de cette dernière fait l'objet d'un « contrat » entre le service social du département, l'association employant les TISF et la famille.

Les entretiens ont été menés par quatre chercheurs dans trois régions avec le même protocole. Nous avons contacté des associations assurant des mesures éducatives d'AEMO qui ont choisi les familles correspondant aux critères communiqués, c'est-à-dire l'intervention simultanée d'une TISF et d'une action éducative. Après avoir demandé l'accord de la famille, les travailleurs sociaux nous ont présentés aux parents. Pour chaque famille, nous avons rencontré séparément au moins trois acteurs (la mère ou les parents, l'éducateur, la TISF) à deux

[9] Bernadette TILLARD, « Observation ethnographique des interactions TISF-Familles », Rapport final PRS 2000-336 Santé des jeunes, Uriopss Nord Pas-de-Calais, 107 p., 2004, http://www.oned.gouv.fr/etudes-recherches-liste-recherches.html).

[10] Bernadette TILLARD «Échanges entre familles et professionnelles. Dons et contre-dons », Ethnologie française, vol. XI, n 1 , pp. 131-139, 2010. 
reprises ; ces deux vagues d'enquête se déroulaient à un an d'intervalle. Au total, 15 cas ont été étudiés dans 3 régions : Rhône-Alpes $\left(\mathrm{A}^{[11]}, \mathrm{B}, \mathrm{C}, \mathrm{D}\right)$, Île-de-France (E, F, H, I, J, K, M) et Nord-Pas-de-Calais (N, O, P). Dans deux services d'AEMO (M, O, P), les TISF faisaient partie de l'équipe éducative. Au total, 79 entretiens ont été menés (23 dans la région RhôneAlpes, 38 en Île-de-France, 18 en Nord-Pas-de-Calais). Ils ont été réalisés au cours des deux périodes d'enquête.

\section{Quelques caractéristiques des familles rencontrées ${ }^{\text {(12) }}$}

Les modalités de mise en relations des chercheurs et des familles ne permettaient pas d'obtenir un échantillon représentatif des familles faisant l'objet d'AEMO. Aussi, est-il utile dans un premier temps de préciser les principales caractéristiques des familles que nous avons rencontrées. Il apparaît que dans 5 cas sur 15 (A, B, F, N, P), les deux parents sont concernés quotidiennement par les tâches éducatives. Fréquemment, un seul parent effectue la majorité des tâches éducatives et de soins vis-à-vis des enfants. Cependant, dans cette étude, il ne s'agit pas seulement de familles monoparentales (E, H, I, J, K, M, O), mais parfois de parents qui, pour différentes raisons telles que l'incarcération (C) ou l'absence du logement adéquat (L), ne vivent pas sous le même toit. Dans deux situations, la charge pèse sur l'un des conjoints en raison de la dépression du partenaire (A) ou de la séparation associée au handicap (D).

Les enfants de ces familles sont d'âges très différents, de 9 mois à 17 ans. Deux enfants uniques font l'objet d'une mesure éducative $(\mathrm{D}, \mathrm{K})$. Parmi les fratries de taille variant de 2 à 8 enfants, les frères et sœurs mineurs cohabitant avec les parents sont tous concernés par l'aide éducative à un moment de l'étude (B, C, E, F, H, I, J, L, M, N, O, P).

Une seule mère $(\mathrm{L})$ travaille à temps partiel (3 h par jour). Toutes les autres mères (seule ou en couple) ne travaillent pas. Globalement, les familles partagent un trait commun : la précarité économique. La seule exception parmi ces 15 familles est une famille de la région Rhône-Alpes (B) dont le père, artisan, possède un emploi stable. Le couple est propriétaire d'une maison individuelle. Toutes les autres familles rencontrées sont locataires. Le cas (B) constitue une double exception puisque, par ailleurs, c'est la seule famille pour laquelle la mesure éducative est de nature administrative et non judiciaire. Au total, les caractéristiques des familles les situent dans une position d'assistance, puisqu'ils sont «dans une relation régulière et contractuelle aux services d'action sociale »[13].

Nous avons choisi de centrer notre propos sur l'effet des changements de domicile des parents sur les relations avec les intervenants sociaux rencontrés. Dans un premier temps, nous envisageons la fréquence de ces déplacements durant notre étude.

[11] Chaque famille ayant fait l'objet d'une étude de cas est désignée par une lettre de l'alphabet de A à P.

[12] Voir le tableau en fin d'article.

[13] Serge PAUGAM, Les formes élémentaires de la pauvreté, Paris, PUF, 2005, pp. 56-57. 


\section{\Familles concernées par les déménagements}

La mobilité familiale observée durant cette enquête ne concerne pas les ménages de la région Rhône-Alpes, mais 6 des 8 familles de la région Île-de-France (F, H, I, J, L, M) et les trois familles de la région Nord-Pas-de-Calais (N, O, P). Étant donné l'absence d'emploi des parents avant et après déménagement, il ne s'agit pas de mobilité dictée par des parcours professionnels, mais plutôt une image du mal-logement des familles précaires en France.

Parfois, la question du logement est à l'origine même de la mesure d'AEMO. Ainsi, pour le cas de la famille $(\mathrm{N})$, l'AEMO a débuté par un épisode qui nous est relaté de manière différente selon les acteurs. Pour l'éducatrice, la famille de 3 enfants à l'époque, était sans logement et a été signalée aux services sociaux parce qu'elle dormait dans un véhicule. Pour la mère, la famille était hébergée par la tante dans un deux-pièces où les parents dormaient dans les fauteuils. La voiturette dont les carreaux étaient cassés était utilisée pour les transports de la famille et a attiré l'attention sur les conditions de transports des enfants. "On vivait à droite, à gauche, chez les gens qui pouvaient nous héberger, mais on vivait pas dans la voiture!»

Certaines familles nombreuses et sans revenu peinent à trouver un logement social adapté à leurs besoins (E, F, I, M, N, O, P). Ainsi, deux familles sont amenées à déménager dans un logement plus grand qui s'avère être insalubre, dans l'attente d'un relogement correct ( $\mathrm{M}$, P). Trois femmes élevant seules leur(s) enfant(s) ont vécu ou vivent (J, L, O) « sans domicile fixe » acceptant de loger chez un membre de la parenté (J), en Centre d'hébergement et de réinsertion sociale (CHRS) $(\mathrm{L})$ ou en foyer maternel $(\mathrm{O})$. La famille J est composée de la mère avec ses trois enfants. La famille est hébergée dans un « hôtel social » en banlieue lointaine où elle occupe un studio de $20 \mathrm{~m}^{2}$, très humide. Le lieu est mal desservi par les transports en commun. À l'entrée de l'immeuble, il n'y a pas de gardien pour accéder au logement. Les conditions sanitaires sont insatisfaisantes. Depuis 3 ans, la mère vit dans des hôtels, passant environ 7 mois dans chaque lieu. La famille vit sans aucune stabilité locative, les contrats d'hébergement sont prolongés tous les 3 mois. L'intervention de la TISF a commencé suite au signalement de la PMI, car l'un des enfants souffrait de la gale. Plus tard, un autre signalement initié par la voisine de l'hôtel, conduit le juge à prononcer une mesure d'AEMO qui, un an et demi après notre rencontre, n'a pas été mise en place. Le signalement concernait le fait que la mère laissait ses enfants seuls durant leur sommeil. Au moment du deuxième entretien, la mère a quitté l'hôtel et n'a plus de relation avec les personnes du réseau social qu'elle s'y était bâti. Elle est hébergée chez sa tante. La TISF qui depuis plus de 2 ans intervient auprès de cette famille, continue son travail chez la tante.

Le mal-logement a des effets directs sur le travail social et la protection de l'enfance. Comme nous l'avons noté, il est parfois à l'origine du suivi éducatif lorsqu'un lieu sûr et pérenne ne peut être garanti par les parents ou leur réseau familial. Lorsque la qualité défectueuse du logement a des conséquences directes sur la santé des enfants (problèmes cutanés, problèmes respiratoires, etc.), ceux-ci alertent les services sanitaires et renforcent le contrôle social. De plus, l'inadaptation du logement engendre une surcharge de travail éducatif pour les parents quand l'organisation de la vie quotidienne ne peut être inventée, tant elle est contrainte par l'étroitesse de l'espace disponible. Enfin, certains logements ne garantissent pas la sécurité domestique, nécessitant un surcroît de vigilance. 
Outre l'accentuation des mesures de protection de l'enfance, les problèmes de logement entretiennent la précarité financière des ménages en raison soit des loyers exorbitants, soit des gouffres financiers que représentent les factures de gaz et d'électricité lorsque le logement est humide et mal isolé. Aussi, certains ménages, qui souhaiteraient s'affranchir d'une tutelle sur les prestations familiales ${ }^{[14]}$, sont obligés d'accepter de composer avec ce qu'ils ressentent comme une intrusion dans leurs choix conjugaux. En contrepartie, ils comptent sur le soutien des travailleurs sociaux pour trouver un logement, répondre aux exigences des propriétaires en matière de garanties, ou encore pour se prémunir d'une expulsion. De plus, ces déménagements successifs, occasionnés par l'insalubrité, engendrent leurs propres coûts financiers de déplacements et de réinstallation.

Ce bilan des déménagements en termes de travail social ou d'économie des ménages ne couvre pas l'ensemble des enjeux de ces déplacements. Trouver l'énergie de faire les paquets, d'organiser la garde des enfants pendant le déménagement, de négocier l'installation de chacun dans un nouvel espace sont le lot commun de toutes les familles qui déménagent, accentué ici par le fait que la précarité économique ne permet pas de déléguer certaines tâches et de s'entourer des soutiens nécessaires. Il s'en suit le fait que les parents vont devoir décider par exemple de changer les enfants d'école en cours d'année ou de compliquer leur quotidien en allant chaque jour à pied dans l'ancien quartier pour y accompagner les enfants. Quelle que soit la solution choisie, elle présente des difficultés. De plus, le déménagement met en péril des réseaux sociaux (voisins, familles) auprès de qui les parents pouvaient trouver du soutien. Cependant, ce dernier élément joue de manière opposée selon que le déménagement permet à la famille de se rapprocher de sa parentèle ou nécessite de s'en éloigner.

80 Compte tenu de leur histoire personnelle, certains de nos interlocuteurs ont une bonne connaissance des travailleurs sociaux. Ces parents d'aujourd'hui ont parfois eux-mêmes vécu la présence de travailleurs sociaux dans leur univers familial durant l'enfance. Leur expérience tient également au placement des aînés de la plupart des fratries (12/15). Parfois, il s'agit d'un placement ayant eu lieu dans le passé (A, D, I, K, P). Pour certains, il s'agit d'un passé proche qui justifie l'intervention actuelle pour faciliter le retour de l'enfant à domicile (A, D). Dans d'autres cas, le placement des aînés se poursuit (F, H, O) et l'AEMO concerne l'éducation des plus jeunes. Pour la famille (F), les travailleurs sociaux s'interrogent sur l'éventualité du placement. Pour la famille (M), un accueil séquentiel ${ }^{[15]}$ des deux enfants qui inclut deux jours et une nuit dans l'institution se verra transformé pour le plus jeune enfant en un placement provisoire complet. Enfin, certains placements sont décidés au cours de la période d'étude (C, K, E, L). En bref, seules trois familles y ont échappé : $\mathrm{B}$, famille la moins précaire et propriétaire de son logement ; $\mathrm{N}$, famille où les problèmes sanitaires et de logement sont plus importants que les problèmes éducatifs, au moins en début d'étude ; et J, famille où la TISF entretient une relation très suivie avec la mère. Selon les professionnels, beaucoup de mesures d'AEMO sont prononcées pour éviter un placement, ou comme alternative au placement. En revanche, pour les parents, derrière une AEMO plane toujours l'ombre d'un placement antérieur et l'éventualité d'un nouveau placement. La

[14] Aujourd'hui administrativement appelée Mesure judiciaire d'aide à la gestion du budget familial (MJAGBF).

[15] Pascale BREUGNOT, Les innovations socio-éducatives. Dispositifs et pratiques innovants dans le champ de la protection de l'enfance, Rennes, Presses de l'EHESP, 2011. Dominique FABLET, « Innover dans le champ de la protection de la suppléance familiale », in Michel CORBILLON (dir.), Suppléance familiale : nouvelles approches, nouvelles pratiques, Vigneux, Matrice, pp. 13-29, 2001. 
présence de quatre nouveaux placements décidés durant l'année parmi les 15 cas de l'étude atteste que leurs craintes correspondent à des pratiques professionnelles relativement fréquentes dans les familles recevant de multiples interventions à domicile.

\section{$\checkmark$ Familles en mourement et services sectorisés : interruptions}

La plupart des services de travail social, qu'ils dépendent du département ou d'une association employant des éducateurs et des TISF, sont organisés en se répartissant l'espace géographique. Si une famille déménage à l'intérieur de la zone desservie par le service d'AEMO, elle garde le même éducateur. Cependant, elle peut très bien changer de lieu de consultation et d'équipe de PMI, ayant franchi la frontière invisible séparant deux espaces desservis chacun par une équipe différente. Les services publics étant en principe destinés à tous alors que le service d'AEMO ne concerne que certains parents, le changement d'équipe de PMI ou d'Assistant(e) social(e) du service social du département est plus fréquent lors d'un déménagement que le changement d'éducateur d'AEMO. Pour ce qui concerne notre étude, étant donné le grand nombre de déménagements et la répartition géographique des services sociaux, le changement d'interlocuteurs des familles est fréquent.

Ces situations créent un espace de liberté pour les familles. Les transmissions de dossiers nécessitent du temps. Les familles ne prenant pas l'initiative de la rencontre, elles peuvent ainsi échapper un moment aux visites des travailleurs sociaux à leur domicile. La sectorisation des services associée au changement résidentiel des familles les soustrait quelques temps aux regards des travailleurs sociaux. Les familles rencontrées font l'objet d'interventions longues de divers services à leur domicile. Les politiques de protection de l'enfance sont encore plus pressantes que le travail social qui encadre les dispositifs de RMI puis de RSA auxquels s'intéressent Serge Paugam et Nicolas Duvoux dans leur dialogue sur La régulation des pauvres ${ }^{[16]}$. En effet, les visites à domicile hebdomadaires ou bihebdomadaires touchent à l'intimité du couple et de la famille. Ce contexte de l'intervention permet de comprendre combien la période du déménagement peut être l'occasion d'expérimenter une phase, fut-elle brève, d'émancipation des suivis dont elles font l'objet. Cette période de latence entre les interventions est perçue comme un moment plutôt agréable pour les familles. En revanche, les travailleurs sociaux mandatés par le juge pour la protection des enfants s'en inquiètent, mais les découvrent a posteriori lors de l'accompagnement de la famille dans son nouveau domicile.

Certes, comme l'ont également relevé Jean-François Laé et Numa Murard, les déménagements sont une manière d'échapper pendant un temps aux relations avec les travailleurs sociaux ${ }^{[17]}$. Cependant, ils ne sont pas le seul facteur créant des interstices. Dans la famille $(\mathrm{N})$, en cours d'étude, l'intervention de la TISF s'interrompt sans que ceci soit réellement planifié. Cindy, la mère, connaît les services sociaux de longue date. Aînée d'une fratrie de 6 enfants, elle a assisté au décès accidentel de son père quand elle avait 12 ans, tout en étant elle-même concernée par l'accident, puisqu'elle était à l'arrière de la mobylette que son père conduisait lorsque tous deux ont été percutés par un véhicule. À la suite du décès paternel, compte tenu de l'état

[16] Serge PAUGAM, Nicolas DUVOUX. La régulation des pauvres. Du RMI au RSA, Paris, PUF, 2008.

[17] Jean-François LAE, Numa MURARD, Deux générations dans la débine. Enquête dans la pauvreté ouvrière, Paris, Bayard, 2011. 
dépressif et de l'alcoolisation de sa propre mère, Cindy et ses frères et sœurs ont été suivis en AEMO durant plusieurs années et deux TISF se sont relayées au domicile de la mère. Lorsque la jeune éducatrice d'AEMO a sollicité les services départementaux pour l'intervention d'une TISF chez Cindy, celle-ci s'est déplacée au siège de l'association de TISF pour demander que ces mêmes TISF interviennent chez elle, de préférence à des professionnelles inconnues. Elle souhaitait renouer les liens antérieurs. Cependant après une période d'intervention d'environ un an, durant laquelle la TISF a participé à l'emménagement de la famille dans son logement, celle-ci a été interrompue. En fait, Cindy qui pourtant entretient avec les TISF des relations jugées satisfaisantes par les deux parties, souhaite que la famille s'affranchisse des différents travailleurs sociaux qui interviennent. Elle ne veut pas bénéficier d'une aide qui se prolonge durant plusieurs années, elle exprime son refus de «toujours dépendre des travailleurs sociaux, comme sa mère ». Aussi, la longue séquence nécessaire au renouvellement de la mesure n'estelle pas entreprise. La démarche à suivre était la suivante : s'adresser à l'assistante sociale de l'UT (Unité territoriale, circonscription du Conseil général), définir avec elle les objectifs de l'intervention, obtenir l'accord pour un certain nombre d'heures d'intervention, puis aller à l'association employant les TISF pour demander la mise en œuvre et payer régulièrement la part qui revient à la famille. Cette procédure n'ayant pas été menée à son terme, l'aide de la TISF s'est interrompue d'elle-même, sans véritablement faire l'objet de relance active de la part de l'AEMO qui a simplement rappelé sans insistance les démarches à la famille. Dans cette situation, la fin de l'activité des TISF durant l'été 2010 coïncide avec la fin de l'emménagement de la famille dans sa nouvelle maison. Supposant une contribution financière des parents, l'intervention nécessite non seulement leur accord de principe, mais également leur collaboration active, leur laissant ainsi un lieu de résistance possible. Par ailleurs, à la suite de ce déménagement, Cindy développe une autre relation privilégiée avec la conseillère en économie sociale et familiale responsable du service de réussite éducative. Celle-ci est intervenue pour que les transports scolaires fassent un détour pour permettre aux enfants de la famille d'utiliser ce service. La relation de Cindy avec cette professionnelle semble remplacer celle entretenue jusque là avec la TISF. Elle la rencontre régulièrement dans les locaux municipaux. Elle accepte et participe avec ses enfants aux propositions de loisirs mises en œuvre par cette professionnelle. La famille ne s'est jamais opposée à la TISF, elle a collaboré un certain temps, puis, sans conflit, a privilégié les relations avec une autre intervenante dont nous pouvons remarquer qu'elle répondait à certains nouveaux besoins des enfants grandissant. Dans la multiplicité des travailleurs sociaux possible, l'éducateur d'AEMO est imposé par le juge, mais la famille garde la possibilité de prendre des initiatives et de privilégier certaines relations parmi les professionnels des autres services sociaux. Ces préférences s'exercent plus particulièrement au moment des recompositions occasionnées par les déménagements.

\section{$\checkmark$ Recompositions des relations après déménagements}

Nous voudrions nous arrêter sur les déménagements successifs d'une même famille $(\mathrm{P})$, car ils illustrent les effets divers de ces déplacements sur les relations de collaboration des partenaires sociaux avec les familles rencontrées durant l'étude. 
Le premier déménagement est motivé par le souhait du couple de se rapprocher des grandsparents paternels. La famille se déplace à $60 \mathrm{~km}$ de son précédent logement. Elle s'installe dans une maison de 3 chambres appartenant à un particulier. Cependant, la pièce principale $\left(12 \mathrm{~m}^{2}\right.$ environ) est de faible surface au regard du nombre d'enfants (5 enfants). La cour de $20 \mathrm{~m}^{2}$ environ comporte un appentis qui tombe en ruine et dont les parents doivent tenir les enfants à distance. Par ailleurs, l'éducatrice assurant la Mesure judiciaire d'aide à la gestion du budget familial de MJAGBF souligne le coût exorbitant du loyer. Dans la ville d'où elle provient, la famille participait au mouvement ATD-Quart Monde. La TISF fréquente également ce mouvement. Son entrée dans la famille en est facilitée. En revanche l'éducatrice d'AEMO et celle en charge de la mesure d'AGBF ont davantage de mal à entrer en relation avec la famille. Ces deux éducatrices trouvent porte close et ne parviennent pas à obtenir la coopération de la famille quant aux pièces administratives nécessaires aux différents dossiers. L'éducatrice d'AEMO appartenant au même service que la TISF, cette dernière facilite progressivement les relations entre la famille et l'éducatrice. D'autre part, le duo « puéricultrice-assistante sociale de circonscription » est très mal perçu par la mère qui trouve ces deux jeunes professionnelles hautaines et méprisantes. Cette attitude est confirmée par la TISF présente au domicile lors d'une de leurs visites et qui à cette occasion n'est ni consultée, ni saluée. Cette situation aboutit au paradoxe d'une relation de la famille plutôt acceptable avec le service d'AEMO mandaté par le juge, alors que le service de droit commun (PMI) est esquivé. Pendant ce temps, le partenariat PMI-AEMO est peu développé. L'éducatrice souligne qu'à plusieurs reprises elle a demandé à rencontrer l'Assistante sociale du département qui ne donne pas suite à la proposition de travailler ensemble sur le copieux dossier qui lui est parvenu de sa collègue éducatrice d'AEMO qui suivait la famille avant le déménagement.

Huit mois plus tard, en raison de l'exiguiité et du coût du logement, la famille déménage de nouveau pour un logement situé à une dizaine de kilomètres du précédent, dans une zone semi-urbaine. La maison, ancienne maison minière vendue à un particulier, est plus spacieuse comportant 3 chambres (6 enfants, un enfant étant né) et un jardin. Elle est située dans le secteur couvert par le service de l'éducatrice AEMO et la TISF. Ces deux professionnelles continuent leur intervention. Un événement majeur survient durant cette année. Il s'agit de la grossesse sous pilule dont Johanna s'est aperçue vers le $4^{\mathrm{e}}$ ou $5^{\mathrm{e}}$ mois, au moment où elle a ressenti les mouvements du fœtus. Cette grossesse permet un rapprochement bien accepté avec la sagefemme de PMI dont la mère apprécie la sollicitude et la compétence. Cette sage-femme lui présente l'équipe locale de PMI (puéricultrice, psychologue et médecin), ce qui permet à la relation de s'instaurer non seulement avec la mère et le nouveau-né, mais également avec le père et les enfants plus âgés. La maman reçoit les visites de l'éducatrice d'AEMO avec laquelle elle collabore a minima, évitant de rentrer en conflit, mais ne donnant pas suite aux conseils prodigués. En satisfaisant aux demandes du médecin et de la puéricultrice en matière sanitaire pour le nouveau-né et en acceptant le suivi psychologique de l'aîné par la psychologue de PMI, elle confie qu'elle espère être bientôt soulagée de la tutelle sur les prestations familiales (MJAGBF). Malgré ces formes de résistance, après ce second déménagement, les relations entre la famille et les travailleurs sociaux sont mieux acceptées. Quelques mois après l'installation, l'éducatrice est informée que la maison avait été déclarée insalubre et qu'elle est louée indument par le propriétaire. La famille déménage à nouveau, cette fois au-delà de la limite du territoire couvert par le service d'AEMO. 
L'exposé de ce cas permet de constater des relations complexes entre la famille et les professionnels des services qui y interviennent : relations parfois fondées sur des valeurs communes (ATD), une expérience quotidienne partagée (TISF), relations évolutives avec les services d'AEMO et de PMI quand une personne parvient à faire reconnaître leurs compétences et à gagner une certaine légitimité aux yeux de la mère (TISF, sage-femme), relation ambivalente acceptée sous contrainte sans réelle collaboration (AEMO), souhait d'émancipation à l'égard de certains travailleurs sociaux (MJAGBF). Les entretiens et les propos informels échangés lors des entretiens ne permettent pas de caractériser de manière définitive et univoque l'attitude de la famille et particulièrement de la mère à l'égard des professionnels. Cette évolution dans le temps des relations entre familles et professionnelles ne permet pas plus de classer chaque famille en caractérisant la forme d'assistance selon les types d'expériences vécues, comme le définit Serge Paugam (différée, installée ou revendiquée). Dans ces familles, l'assistance est imposée pour ce qui concerne l'assistance éducative en milieu ouvert et la mesure judiciaire d'aide à la gestion du budget familial, toutes deux mandatées par le juge des enfants. Pour ce qui concerne les autres services autour de la famille, ils sont tantôt vécus comme « assistance différée », tantôt « assistance installée », selon les moments et selon les services. Cette complexité des relations diverses et fluctuantes avec les partenaires ne peut être définie par la seule alternative opposition/ collaboration. Étant donné la multiplicité des interventions auprès des familles et le degré varié de collaboration et de résistance, la métaphore qui s'impose est celle d'une mosaïque de relations aux tonalités variées. Chaque déménagement intervient alors comme un moment particulier qui vient provoquer une recomposition du puzzle des relations.

Dans le cas précédent, le second déménagement est associé à une meilleure collaboration. Inversement, les changements de secteurs et certains défauts de circulation d'informations conduisent parfois à des conflits. Ainsi, dans le cas (J), le conflit avec l'assistante sociale portait sur le fait que la mère devait faire suivre son enfant dans un service de PMI de la circonscription de l'assistante sociale, alors que celui-ci était très éloigné de son nouveau lieu de résidence. La mère refusait de s'y rendre (coût de transport) et faisait soigner ses enfants dans un centre de santé situé près de son hôtel. Le non-respect de l'injonction de l'assistante sociale aurait pu être considéré comme un facteur aggravant la situation des enfants. Cependant, l'intervention de la TISF qui informe le service social de département que la mère prend soin de son enfant et le fait suivre dans un endroit différent de celui prescrit, évite que ce refus soit considéré comme une négligence. Ici, comme précédemment, la TISF joue un rôle de médiation entre les services sociaux et la famille.

Un des avantages de cette sectorisation est de pouvoir retrouver un(e) professionnel(le) connu(e) quand les familles reviennent près des grands-parents maternels ou paternels. Ce fut le cas précédemment cité où Cindy retrouve les TISF qu'elle a connues durant son enfance. Réciproquement, cela permet au médecin de PMI de connaître Colette $(\mathrm{O})$ et toute sa famille, évitant à celle-ci de longs développements sur les tribulations familiales. Elle apprécie également d'avoir toujours la même éducatrice d'AEMO comme interlocutrice. Quand les relations sont bonnes, cela semble donc une économie relationnelle intéressante pour les parents comme pour les travailleurs sociaux. Étant donné que les parents ne choisissent pas leurs travailleurs sociaux ou leurs équipes de PMI comme ils ont la possibilité de le faire pour les professionnels libéraux, si les relations sont fondées sur la confiance et la reconnaissance d'un certain degré de légiti- 
mité ${ }^{[18]}$, elles engendrent certaines collaborations. Au contraire, quand des conflits éclatent entre les services sociaux et les parents, ces derniers doivent composer avec la contrainte de cette relation mandatée par le juge, jusqu'à l'éventuel changement d'affectation de l'éducateur ou jusqu'au prochain déménagement. Ce déplacement résidentiel s'accompagne d'une période de flottement suivie d'une réorganisation des relations qui prennent la forme de résistance, d'affinités, de conflit ou d'acceptation sans collaboration active.

\section{$\checkmark$ Conclusion}

Les familles étudiées ont des trajectoires résidentielles complexes. Leur mobilité n'est pas une mobilité économique qui les conduirait à se déplacer pour trouver ou garder un emploi. De plus, étant donné l'absence de capital immobilier, la mobilité des familles ne ralentit pas avec l'âge des parents, comme cela a été observé dans d'autres classes sociales. Ces changements de logement sont plutôt des adaptations successives : d'une part ; aux propositions de structures d'accueil (CHRS, foyer maternel, hôtel social) et au manque de logement social adapté[ ${ }^{[19]}$; d'autre part, aux besoins changeant du fait de la taille de la famille et des aléas conjugaux. De plus, lors de ces déplacements, la famille élargie est prise en compte comme cela a été observé dans les travaux de Catherine Bonvalet ${ }^{[20]}$. Dans notre étude, les familles se rapprochent des grands-parents maternels ou paternels ou d'un enfant plus âgé installé en couple ou encore de l'établissement dans lequel leurs enfants ont été placés. Elles se déplacent sur des distances variables (de quelques $\mathrm{km}$ à $60 \mathrm{~km}$ ) et s'installent dans des lieux connus au moins par un membre du couple. Dans leur étude à Elbœuf, Jean-François Laé et Numa Murard parlent d'une «mobilité permanente » qui « reste confinée dans le cercle de la commune, incluant au mieux les communes voisines ${ }^{[21]}$. Cependant dans notre cas, les familles situées dans deux régions très urbanisées, se déplacent sur une zone plus étendue. Même si le réseau familial est pris en compte dans ces déplacements, il ne semble pas approprié de parler de « captivité résidentielle » ${ }^{[22]}$, mais plutôt de précarité résidentielle. Si les travaux attribuent généralement aux familles pauvres une moins grande mobilité, nous observons ici une sorte de mobilité paradoxale.

Cette étude renvoie à une dimension essentielle de l'éducation de l'enfant dans sa famille, celle du logement. D'autres études ont souligné cette importance. Ainsi, les travaux de Bruce Leslie au Canada montrent que parmi les enfants pris en charge par la protection de l'enfance, pour un enfant sur cinq, le logement est pris en compte dans la décision, qu'elle soit d'admettre l'enfant dans un service, ou de retarder son retour en famille ${ }^{[23]}$. Plus proches de notre recherche, les premiers résultats du travail doctoral d'Amélie Turlais mené sur les décisions du juge des

[18] Dominique FABLET, Marie-Pierre MACKIEWICZ, «Les modalités de coopération entre professionnels et parents d'enfants placés dans les pouponnières à caractère social », Rapport pour la MIRE/DEP, convention 17/93, 1996.

[19] Nicolas DUVOUX, Le nouvel âge de la solidarité. Pauvreté, précarité et politiques publiques, Paris, Seuil, 2012, p. 101.

[20] Catherine BONVALET, « Sociologie de la famille, sociologie du logement : un lien à redéfinir », Sociétés Contemporaines, $\mathrm{n}^{\circ} 25,1997$, pp. 25-44.

[21] Jean-François LAE, Numa MURARD, Deux générations dans la débine. Enquête dans la pauvreté ouvrière, Paris, Bayard, 2011, p. 46.

[22] Sylvie FOL, « Encouragement ou injonction à la mobilité ? », Projet, n 314, 2010, pp. 52-58.

[23] Bruce LESLIE, « Housing influences on child welfare: A practice response with service and policy implications », in Jane SCOTT, Harriet WARD (eds), Safeguarding and Promoting the Well-being of Children, Families and Communities. London, Jessica Kingsley Publishers, 2005, pp. 213-227. 
enfants à l'issue d'une mesure d'AEMO nous indiquent que dans $16 \%$ des cas, le juge n'est plus concerné par la mesure en raison d'un changement de juridiction de l'enfant ${ }^{[24]}$. Notre étude, centrée sur des familles recevant plusieurs prestations à domicile, grâce à son organisation en deux périodes, met en évidence le poids du logement dans 9 cas 15 . La précarité économique des familles associée à l'insalubrité des logements qui leur sont accessibles conduit à des ruptures d'interventions suivies de recomposition. Cette conclusion conduit indirectement à interroger l'organisation distincte et peu connectée des politiques de rénovation urbaine, relevant de l'État et des municipalités, et des politiques de protection de l'enfance, relevant du département et du ministère la Justice.

[24] Amélie TURLAIS, Paul DURNING, « Maintaining or removing children from their family environment : a study of influences on judiciairy décisions after a home-based intervention », Communication, $12^{\text {th }}$ International Conference of EUSARF, Glasgow, 4-7 sept. 2012, p. 153. 
Tableau synoptique des enquêtés

\begin{tabular}{|c|c|c|c|c|c|c|c|c|}
\hline & \begin{tabular}{|c|} 
Âge \\
enfants
\end{tabular} & AEMO & Père & Mère & Placements & $\begin{array}{l}\text { Autres ruptures } \\
\text { ou difficultés }\end{array}$ & Continuité & Intervenants \\
\hline A & $\begin{array}{l}21 \text { ans, } \\
18 \text { ans, } \\
9 \text { mois }\end{array}$ & 9 mois & $\begin{array}{l}\text { Entre } \\
\text { chômage et } \\
\text { intérim dans } \\
\text { le bâtiment }\end{array}$ & $\begin{array}{l}\text { Sans travail, } \\
\text { dépression } \\
\text { post-partum }\end{array}$ & $\begin{array}{l}\text { Placement du bébé } \\
\text { durant } 3 \text { mois, } \\
\text { Hospitalisation } \\
\text { de la mère en } \\
\text { psychiatrie }\end{array}$ & $\begin{array}{l}\text { Fin brutale du } \\
\text { dispositif d'AEMO } \\
\text { renforcée entraînant } \\
\text { le changement } \\
\text { d'éducateur et de } \\
\text { service concerné }\end{array}$ & $\begin{array}{l}\text { Intervention de la } \\
\text { TISF, le couple } \\
\text { et la composition } \\
\text { familiale ( } 2 \text { aînés } \\
\text { présents), le } \\
\text { logement HLM } \\
\text { dans une commune } \\
\text { périurbaine }\end{array}$ & $\begin{array}{l}\text { AEMO, } \\
\text { TISF, ASE, } \\
\text { CMP, PMI }\end{array}$ \\
\hline B & $\begin{array}{l}13 \text { ans, } \\
8 \text { ans }\end{array}$ & $\begin{array}{l}13 \text { ans, } \\
8 \text { ans }\end{array}$ & Artisan & Au foyer & $\begin{array}{l}\text { Peur du placement } \\
\text { exprimée par les } \\
\text { parents (demande } \\
\text { initiale d'aide ; aide } \\
\text { reçue en décalage } \\
\text { par rapport à leurs } \\
\text { attentes) }\end{array}$ & $\begin{array}{l}\text { difficultés scolaires } \\
\text { des enfants, la mère } \\
\text { se plaint de son } \\
\text { manque d'autorité } \\
\text { et de son isolement. }\end{array}$ & $\begin{array}{l}\text { Travail du père, } \\
\text { artisan. Le } \\
\text { logement de la } \\
\text { famille propriétaire } \\
\text { d'une vaste villa en } \\
\text { milieu campagnard }\end{array}$ & AED, TISF \\
\hline $\mathrm{C}$ & $\begin{array}{l}15 \text { ans, } \\
14 \text { ans, } \\
12 \text { ans, } \\
11 \text { ans, } \\
7 \text { ans }\end{array}$ & $\begin{array}{l}15 \text { ans, } \\
14 \text { ans, } \\
12 \text { ans, } \\
11 \text { ans, } \\
7 \text { ans }\end{array}$ & Incarcéré & $\begin{array}{l}\text { Sans emploi, } \\
\text { bénévole } \\
\text { d'un club de } \\
\text { sport }\end{array}$ & $\begin{array}{l}\text { Placement de } \\
\text { l'aînée pendant } \\
\text { l'étude }\end{array}$ & $\begin{array}{l}\text { Père incarcéré suite } \\
\text { à abus sexuel sur } \\
\text { une de ses filles. } \\
\text { Emménagement en } \\
\text { appartement HLM } \\
\text { d'une banlieue } \\
\text { de grande ville. } \\
\text { Violence entre les } \\
\text { enfants. } \\
\end{array}$ & $\begin{array}{l}\text { Changement } \\
\text { d'éducatrice } \\
\text { spécialisée en } \\
\text { cours de mesure. } \\
\text { Changement } \\
\text { de TISF à deux } \\
\text { reprises entre } \\
18 \text { mois }\end{array}$ & $\begin{array}{l}\text { AEMO, } \\
\text { TISF, AGBF }\end{array}$ \\
\hline D & 8 ans & 8 ans & $\begin{array}{l}\text { Sans travail, } \\
\text { bénéficiaire } \\
\text { de l'AAH }\end{array}$ & $\begin{array}{l}\text { Sans } \\
\text { profession, } \\
\text { non } \\
\text { concernée } \\
\text { par l'AEMO }\end{array}$ & $\begin{array}{l}\text { Placement de } \\
\text { l'enfant en famille } \\
\text { d'accueil pendant } \\
4 \text { ans }\end{array}$ & $\begin{array}{l}\text { Accident ayant } \\
\text { provoqué une } \\
\text { réduction } \\
\text { des facultés } \\
\text { intellectuelles } \\
\text { du père (ne sait } \\
\text { plus compter). } \\
\text { Divorce du couple. } \\
\text { Hospitalisation } \\
\text { du père pour } \\
\text { dépression en cours } \\
\text { d'étude. }\end{array}$ & & $\begin{array}{l}\text { AEMO, } \\
\text { TISF au } \\
\text { domicile du } \\
\text { père }\end{array}$ \\
\hline $\mathrm{E}$ & $\begin{array}{l}16 \text { ans, } \\
16 \text { mois }\end{array}$ & 16 mois & Mère seule & Sans travail & $\begin{array}{l}\text { Placement de } \\
\text { la jeune fille } \\
\text { durant l'étude, de } \\
\text { nouveau fugue, } \\
\text { suivie de «main } \\
\text { levée de l'OPP » } \\
\text { (Ordonnance } \\
\text { Provisoire de } \\
\text { Placement) } \\
\end{array}$ & $\begin{array}{l}\text { Fugues de la jeune } \\
\text { fille, refus de } \\
\text { l'AEMO. Parcours } \\
\text { migratoire de la } \\
\text { mère : Cameroun, } \\
\text { Sénégal, France. A } \\
\text { connu différentes } \\
\text { périodes d'activité } \\
\text { salariée. }\end{array}$ & $\begin{array}{l}\text { Appartement F2 } \\
\text { HLM depuis } 2001 .\end{array}$ & $\begin{array}{l}\text { AEMO, } \\
\text { TISF, CESF, } \\
\text { CMP (pour } \\
\text { la mère) }\end{array}$ \\
\hline $\mathrm{F}$ & $\begin{array}{l}18 \text { ans, } \\
16 \text { ans, } \\
6 \text { ans, } \\
3 \text { ans }\end{array}$ & 3 ans & Sans travail & Sans travail & $\begin{array}{l}\text { Placement actuel } \\
\text { des } 3 \text { aînés de la } \\
\text { fratrie dans des } \\
\text { lieux différents. } \\
\text { Placement } \\
\text { envisagé pour le } \\
\text { plus jeune durant } \\
\text { toute l'étude. }\end{array}$ & $\begin{array}{l}\text { Emménagement } \\
\text { récent dans un } \\
\text { HLM } 4 \text { pièces. } \\
\text { Changement } \\
\text { de TISF car la } \\
\text { première tentative } \\
\text { d'intervention a été } \\
\text { un échec }\end{array}$ & $\begin{array}{l}\text { Parents présentés } \\
\text { comme « déficients } \\
\text { intellectuels » par } \\
\text { les travailleurs } \\
\text { sociaux }\end{array}$ & $\begin{array}{l}\text { AEMO, } \\
\text { TISF, CMP } \\
\text { (mère). } \\
\text { Deux } \\
\text { familles } \\
\text { d'accueil. }\end{array}$ \\
\hline
\end{tabular}


Tableau synoptique des enquêtés (suite)

\begin{tabular}{|c|c|c|c|c|c|c|c|c|}
\hline & \begin{tabular}{|c|}
$\begin{array}{c}\text { Âge } \\
\text { enfants }\end{array}$ \\
\end{tabular} & AEMO & Père & Mère & Placements & $\begin{array}{c}\text { Autres ruptures } \\
\text { ou difficultés }\end{array}$ & Continuité & Intervenants \\
\hline $\mathrm{H}$ & \begin{tabular}{|l|l}
5 \\
enfants
\end{tabular} & 2 ans & Mère seule & \begin{tabular}{|l} 
Femme sans \\
travail
\end{tabular} & $\begin{array}{l}\text { Placement des } \\
\text { quatre aînés de la } \\
\text { fratrie. Violence } \\
\text { de chaque parent } \\
\text { envers les enfants. }\end{array}$ & \begin{tabular}{|l|} 
Parcours migratoire \\
des parents. \\
Séparation du \\
couple parental. \\
Déménagement \\
pour un \\
appartement plus \\
petit.
\end{tabular} & $\begin{array}{l}\text { Soupçon de } \\
\text { persistance } \\
\text { de violences } \\
\text { maternelles à } \\
\text { l'égard de l'enfant }\end{array}$ & \begin{tabular}{|l} 
AEMO, \\
TISF
\end{tabular} \\
\hline I & $\begin{array}{l}14 \text { ans, } \\
11 \text { ans, } \\
6 \text { ans, } \\
4 \text { ans }\end{array}$ & $\begin{array}{l}14 \text { ans, } \\
11 \text { ans, } \\
6 \text { ans, } \\
4 \text { ans }\end{array}$ & Mère seule & Sans travail & $\begin{array}{l}\text { Antécédent de } \\
\text { placement des } \\
2 \text { aînés. À sa } \\
\text { demande, la fille } \\
\text { aînée entre en } \\
\text { internat durant } \\
\text { l'enquête. }\end{array}$ & \begin{tabular}{|l|} 
Changement \\
d'appartement \\
pour un F4 dans la \\
même cité (en lien \\
avec la constitution \\
d'un nouveau \\
couple pendant la \\
recherche). Mère \\
impulsive, « élève \\
la voix sur les \\
enfants!» \\
\end{tabular} & $\begin{array}{l}\text { Fin de la mesure de } \\
\text { l'intervention sans } \\
\text { information à la } \\
\text { TISF. C'est la mère } \\
\text { qui la prévient. }\end{array}$ & $\begin{array}{l}\text { AEMO, } \\
\text { TISF }\end{array}$ \\
\hline $\mathrm{J}$ & $\begin{array}{l}7 \text { ans, } \\
5 \text { ans, } \\
3 \text { ans }\end{array}$ & $\begin{array}{l}7 \text { ans, } \\
5 \text { ans, } \\
3 \text { ans }\end{array}$ & Mère seule & Sans travail & & \begin{tabular}{|l|} 
Parcours \\
migratoire. De \\
chambre d'hôtel en \\
studio insalubre. \\
Hébergement en fin \\
d'étude chez une \\
tante. Avortement \\
en cours d'étude. \\
Demande de \\
changer d'AS. \\
Conflit avec la PMI \\
(éloignement, gale).
\end{tabular} & $\begin{array}{l}\text { TISF assure la } \\
\text { continuité du suivi, } \\
\text { joue la médiatrice } \\
\text { avec l'ASE et la } \\
\text { PMI }\end{array}$ & \begin{tabular}{|l|} 
AEMO, \\
TISF, PMI, \\
CMP (enfant \\
â̂né + mère), \\
pôle emploi \\
\end{tabular} \\
\hline $\mathrm{K}$ & 11 ans & 11 ans & Mère seule & Sans travail & $\begin{array}{l}\text { Antécédent de } \\
\text { placement de } \\
\text { l'enfant à } 3 \text { ans } \\
\text { durant plusieurs } \\
\text { années. Durant } \\
\text { l'étude, départ } \\
\text { pour l'internat } \\
\text { scolaire et reprise } \\
\text { du placement } \\
\text { « séquentiel ». }\end{array}$ & \begin{tabular}{|l|} 
Rupture de la \\
mère avec ses \\
parents à l'âge de \\
18 ans. Arrêt de \\
l'intervention de la \\
TISF quand l'enfant \\
entre en internat. \\
Premier garçon \\
dont elle est sans \\
nouvelle depuis que \\
son compagnon est \\
parti avec l'enfant \\
\end{tabular} & \begin{tabular}{|l|} 
L'assistante \\
familiale (famille \\
d'accueil) \\
concernée par \\
l'accueil de \\
l'enfant est \\
toujours la même.
\end{tabular} & \begin{tabular}{|l} 
AEMO, \\
TISF, \\
Assistante \\
familiale
\end{tabular} \\
\hline $\mathrm{L}$ & \begin{tabular}{|l|}
14 ans, \\
12 ans, \\
nné
\end{tabular} & $\begin{array}{l}14 \text { ans, } \\
12 \text { ans, } \\
\text { nné }\end{array}$ & Mère seule & Sans travail & $\begin{array}{l}\text { Le fils aîné a été } \\
\text { "placé en internat » } \\
\text { pour le soustraire } \\
\text { à la violence } \\
\text { paternelle. }\end{array}$ & $\begin{array}{l}\text { Parcours migratoire } \\
\text { de la mère (origine } \\
\text { Cameroun). } \\
\text { Séparation du } \\
\text { couple. La mère } \\
\text { vit en studio en } \\
\text { CHRS. Interruption } \\
\text { de l'intervention } \\
\text { de la TISF } \\
\text { (constat partagé } \\
\text { d'inefficacité). }\end{array}$ & & $\begin{array}{l}\text { AEMO, } \\
\text { TISF }\end{array}$ \\
\hline
\end{tabular}


Tableau synoptique des enquêtés (suite)

\begin{tabular}{|c|c|c|c|c|c|c|c|c|}
\hline & \begin{tabular}{c|} 
Âge \\
enfants
\end{tabular} & AEMO & Père & Mère & Placements & $\begin{array}{c}\text { Autres ruptures } \\
\text { ou difficultés }\end{array}$ & Continuité & Intervenants \\
\hline $\mathrm{M}$ & $\begin{array}{l}22 \text { ans, } \\
11 \text { ans, } \\
9 \text { ans }\end{array}$ & $\begin{array}{l}11 \text { ans, } \\
9 \text { ans }\end{array}$ & Mère seule & Sans travail & $\begin{array}{l}\text { «Placement } \\
\text { séquentiel » de } \\
\text { l'enfant de } 9 \text { ans. }\end{array}$ & $\begin{array}{l}\text { Parcours migratoire } \\
\text { des parents } \\
\text { (origine Algérie). } \\
\text { Incarcération du } \\
\text { père durant la } \\
\text { première grossesse. } \\
\text { Divorce avec } \\
\text { violence conjugale. } \\
\text { Mère déménage } \\
\text { d'un petit logement } \\
\text { à un logement } \\
\text { plus grand mais } \\
\text { insalubre, en } \\
\text { attente de } \\
\text { relogement. }\end{array}$ & & $\begin{array}{l}\text { AEMO, } \\
\text { TISF, CMP, } \\
\text { Secteur, AS } \\
\text { école, } \\
\text { accueil } \\
\text { séquentiel... }\end{array}$ \\
\hline $\mathrm{N}$ & \begin{tabular}{|l}
6 ans, \\
4 ans, \\
3 ans, \\
18 mois
\end{tabular} \mid & $\begin{array}{l}6 \text { ans, } \\
4 \text { ans, } \\
3 \text { ans, } \\
18 \text { mois }\end{array}$ & $\begin{array}{l}\text { Père entre } \\
\text { chômage et } \\
\text { intérim }\end{array}$ & Sans travail & $\begin{array}{l}\text { La mère a connu } \\
\text { une mesure } \\
\text { d'AEMO à } \\
\text { l'adolescence après } \\
\text { le décès paternel } \\
\text { et dépression } \\
\text { maternelle. }\end{array}$ & $\begin{array}{l}\text { Emménagement } \\
\text { récent après années } \\
\text { d'hébergement } \\
\text { par différents } \\
\text { membres de la } \\
\text { famille. Maladie } \\
\text { orpheline du père } \\
\text { et de } 3 \text { des enfants. } \\
\text { Changement } \\
\text { d'éducatrice } \\
\text { d'AEMO, fin de } \\
\text { l'intervention de la } \\
\text { TISF. }\end{array}$ & $\begin{array}{l}\text { TISF identique à } \\
\text { celle connue par } \\
\text { la mère durant } \\
\text { l'enfance, relais par } \\
\text { la CESF du PRE }\end{array}$ & $\begin{array}{l}\text { AEMO, } \\
\text { TISF, PRE, } \\
\text { PMI, CHRU, } \\
\text { AGB }\end{array}$ \\
\hline $\mathrm{O}$ & $\begin{array}{l}26 \text { ans, } \\
25 \text { ans, } \\
20 \text { ans, } \\
18 \text { ans, } \\
13 \text { ans, } \\
12 \text { ans, } \\
7 \text { ans, } \\
\text { nné }\end{array}$ & $\begin{array}{l}13 \text { ans, } \\
12 \text { ans, } \\
7 \text { ans, } \\
\text { nné }\end{array}$ & Mère seule & Sans travail & $\begin{array}{l}\text { Trois enfants } \\
\text { mineurs placés en } \\
\text { MECS durant la } \\
\text { majeure partie de } \\
\text { l'étude. }\end{array}$ & $\begin{array}{l}\text { Incarcération de } \\
\text { l'aîné et de l'oncle } \\
\text { maternel pour abus } \\
\text { sexuel sur certains } \\
\text { enfants de la fratrie. } \\
\text { Quatre lieux de vie } \\
\text { différents pour la } \\
\text { mère durant } \\
\text { l'étude. Survenue } \\
\text { d'une maladie } \\
\text { grave en fin } \\
\text { d'étude. }\end{array}$ & $\begin{array}{l}\text { Persistance } \\
\text { de rencontres } \\
\text { hebdomadaires } \\
\text { à la MECS, } \\
\text { retrouvailles avec } \\
\text { la même TISF, } \\
\text { maintien de la } \\
\text { même ES }\end{array}$ & $\begin{array}{l}\text { AEMO, } \\
\text { TISF, PMI, } \\
\text { Secteur, } \\
\text { AGBF, } \\
\text { Foyer } \\
\text { maternel, } \\
\text { etc. }\end{array}$ \\
\hline $\mathrm{P}$ & \begin{tabular}{|l}
13 ans, \\
9 ans, \\
6 ans, \\
5 ans, \\
4 ans, \\
2 ans, \\
1 an \\
\end{tabular} & $\begin{array}{l}6 \text { ans, } \\
5 \text { ans, } \\
4 \text { ans, } \\
2 \text { ans, } \\
1 \text { an }\end{array}$ & $\begin{array}{l}\text { Père entre } \\
\text { chômage et } \\
\text { intérim }\end{array}$ & Sans travail & $\begin{array}{l}\text { Antécédent de } \\
\text { placements des } \\
\text { enfants durant un } \\
\text { épisode de rupture } \\
\text { conjugale. Les } \\
\text { deux aînés de la } \\
\text { mère perdus de vue } \\
\text { vivent avec leur } \\
\text { père. }\end{array}$ & $\begin{array}{l}2 \text { logements } \\
\text { différents en cours } \\
\text { d'étude, le } 2^{\mathrm{e}} \\
\text { étant insalubre. } \\
\text { Naissance en } \\
\text { cours d'étude, } \\
\text { changement } \\
\text { d'équipe de PMI } \\
\text { (déménagement). }\end{array}$ & & $\begin{array}{l}\text { AEMO, } \\
\text { TISF, PMI, } \\
\text { AGBF }\end{array}$ \\
\hline
\end{tabular}

